\title{
COMMENTARY
}

\section{The thermodynamic brain}

\author{
Joseph Donnelly ${ }^{1}$ and Marek Czosnyka ${ }^{1,2^{*}}$ \\ See related research by Stretti et al., http://ccforum.com/content/18/5/552
}

\begin{abstract}
Apart from its complex functionality, the brain is a robust thermodynamic machine; the tissue metabolic rate is high and it is thermally shielded by a skull. Therefore, if there is no high-volume blood flow to cool and stabilize the brain temperature, the possibility of unstable behavior seems to be high. Inflowing arterial blood is normally cooler than the brain tissue temperature, and outflowing venous blood is normally warmer than arterial blood but cooler than the brain tissue. Brain blood flow can thus be understood as a cooler for the brain. Pros and cons of clinical measurement, with clear indication for a multimodal monitoring approach, are discussed along with a brief review of basic facts known about temperature, cerebral blood flow and volume, intracranial pressure, and compartmental compliances of the brain.
\end{abstract}

A large quantity of experimental works, sometimes with conflicting conclusions, have been published about the regulation of core temperature and brain temperature. Bedside clinical studies, however, are not that common. Measurement of cerebral blood flow in comparison with core body temperature in traumatic brain injury (TBI) patients, as presented in a recent issue of Critical Care by Stretti and colleagues, is therefore worth commendation [1].

Cooling the body to protect the brain and preserve life may sound like something from science fiction. However, cooling seems to have very real beneficial effects. Some of our friends in the animal kingdom can withstand remarkable physiologic insults, at least when they are cold. For example, Spermophilus tridecemlineatus (ground squirrels) can tolerate $90 \%$ reductions in cerebral perfusion

\footnotetext{
* Correspondence: mc141@medschl.cam.ac.uk
${ }^{1}$ Division of Neurosurgery, Department of Clinical Neurosciences,

* Correspondence: mc141@medschl.cam.ac.uk
${ }^{1}$ Division of Neurosurgery, Department of Clinical Neurosciences,

Addenbrooke's Hospital, University of Cambridge, Hills Road, Cambridge CB2

$\mathrm{OQQ}$, UK
${ }^{2}$ Institute of Electronic Systems, Warsaw University of Technology,

${ }^{2}$ Institute of Electronic Systems, Warsaw University of Technology, Nowowiejska 15/19, Warsaw, Poland
}

(c) 2014 Donnelly and Czosnyka; licensee BioMed Central Ltd. The licensee has exclusive rights to distribute this article, in any medium, for 12 months following its publication. After this time, the article is available under the terms of the Creative Commons Attribution License (http://creativecommons.org/licenses/by/4.0), which permits unrestricted use, distribution, and reproduction in any medium, provided the original work is properly credited. The Creative Commons Public Domain Dedication waiver (http://creativecommons.org/publicdomain/zero/1.0/) applies to the data made available in this article, unless otherwise stated. without any neurologic deficit, provided their temperature is reduced to $10^{\circ} \mathrm{C}$ during hibernation [2]. In fact, cooling has been used as a therapy in medicine for decades [3]. Since its inception, enthusiasm for cooling has waxed and waned in the domains of cardiopulmonary bypass, cardiac arrest, stroke, and TBI [4]. Because temperature has the potential to alter cerebral metabolism, blood flow and intracranial pressure (ICP), therapeutic cooling has been proffered as a management strategy after TBI. However, clinical trials in TBI have not been conclusive to date $[5,6]$, prompting a new large-scale multicenter European trial [7]. In any case, clinical trials usually differ from scientific procedures because they provide pragmatic answers for clinicians (if conclusive) but often not for scientists. The need to establish a link between clinical utility and scientific rationale forces us to return to simple questions such as that posed by Stretti and colleagues: what are the brain hemodynamic effects of temperature changes?

The basic tenants underpinning therapeutic cooling in TBI are related to the fundamental relationship between temperature and the rate of biochemical reactions common to all species [8], and the effect of temperature on ICP. The brain may be particularly sensitive to changes in temperature for two reasons: the brain is highly metabolically active; and, due to the rigid cranium, temperature-induced changes in metabolism and cerebral blood volume can result in changes in ICP. The ICP itself is governed by the volume of the various compartments in the skull; namely, the vascular, parenchymal, and cerebrospinal fluid (CSF) compartments. The question then arises as to which component of ICP temperature affects.

The vascular component is the obvious choice because decreasing temperature increases vascular tone in the small pial vessels [9], and perhaps even in the basal arteries [10]. Aside from altering the vascular component of ICP, it is also possible (and as yet unknown) that the CSF or parenchymal compartments are altered - for example, by altering CSF production or reabsorption, or by affecting the osmotic composition of the parenchymal interstitium. 
Separating such components will be difficult to achieve experimentally and especially clinically.

Stretti and colleagues used transcranial Doppler (TCD) ultrasonography during alteration in body temperature after TBI in an attempt to further our understanding of the cerebral hemodynamic consequences of cooling. TCD is a stethoscope for the brain, and its bedside use in several scenarios should be more widespread. The advantages of TCD measurements on arrival at neurocritical care, to make a quick assessment and decide about the first few hours' management strategy, have been highlighted recently [11]. TCD measures blood flow velocity, not volumetric flow, and because it has a pulsatile component can (and should) be analyzed with pulse waveform signal processing methodology. In addition, combining TCD velocity measures with arterial blood pressure and cerebral perfusion pressure can provide further insight into cerebral hemodynamics by describing the autoregulation, vascular compliance, resistance, time constant, wall tension, critical closing pressure, and other parameters.

In the current study, the relationships between core body temperature and cerebral hemodynamics were studied in two groups of TBI patients: those with a fever who were subsequently cooled (defervescence group); and those who were hypothermic who were warmed to normothermia (rewarming group) [1]. The mean flow velocity observed in the rewarming group is nearly twice lower than that in the defervescence group. This disproportion remains apparent even if the upper temperature in rewarmed patients was close to the lower temperature in the defervescence group. This observation may suggest that there is no one universal temperaturecerebral blood flow relationship, and other physiological variables obviously play a role. The authors report that with lower temperature we see lower mean arterial pressure and lower TCD pulsatility index. This is a novel finding. The pulsatility index is theoretically proportional to the pulsation of blood pressure and (nonlinearly) to a product of cerebrovascular resistance and arterial compartmental compliance multiplied by the heart rate, but inversely proportional to cerebral perfusion pressure [12]. We still know little about vascular resistance and compliance when temperature varies, and cerebral perfusion pressure is reported to stay constant at least in the defervescence group; therefore, it is possible that the lowering of arterial blood pressure pulse amplitude is responsible for the reduced pulsatility index with lower temperature.

In conclusion, Stretti and colleagues touch complex and still poorly chartered phenomena. Because of the multifactorial interactions between brain injury, temperature control, cerebral blood flow, and autoregulation, the answer to all questions is impossible within an observational study design based on a limited number of cases. Nevertheless, this paper opens a thought-provoking discussion and, we hope, will stimulate further clinical research in the area of the thermodynamic brain.

\section{Abbreviations \\ CSF: Cerebrospinal fluid; ICP: Intracranial pressure; TBI: Traumatic brain injury; TCD: Transcranial Doppler.}

\section{Competing interests}

The authors declare that they have no competing interests.

\section{Acknowledgments}

MC is supported by the NIHR Biomedical Research Centre in Cambridge, UK and JD is supported by a Woolf Fisher Scholarship.

Published online: 12 December 2014

\section{References}

1. Stretti F, Gotti M, Pifferi S, Brandi G, Annoni F, Stocchetti N: Body temperature affects cerebral hemodynamics in acutely brain injured patients: an observational transcranial color-coded duplex sonography study. Crit Care 2014, 18:552.

2. Frerichs KU, Kennedy C, Sokoloff L, Hallenbeck JM: Local cerebral blood flow during hibernation, a model of natural tolerance to 'cerebral ischemia'. J Cereb Blood Flow Metab 1994, 14:193-205.

3. Fay T: Cooling in shock. J Am Med Assoc 1943, 121:1109.

4. Mrozek S, Vardon F, Geeraerts T: Brain temperature: physiology and pathophysiology after brain injury. Anesthesiol Res Pract 2012, 2012:1-13.

5. Clifton GL, Miller ER, Choi SC, Levin HS, McCauley S, Smith KR, Muizelaar JP, Wagner FC, Marion DW, Luerssen TG, Chesnut RM, Schwartz M: Lack of effect of induction of hypothermia after acute brain injury. $N$ Engl I Med 2001, 344:556-563.

6. Peterson K, Carson S, Carney N: Hypothermia treatment for traumatic brain injury: a systematic review and meta-analysis. J Neurotrauma 2008, 25:62-71.

7. Eurotherm 3235 Trial. [http://www.eurotherm3235trial.eu/home/index.phtml]

8. Gillooly JF, Brown JH, West GB, Savage VM, Charnov EL: Effects of size and temperature on metabolic rate. Science 2001, 293:2248-2251.

9. Ueda Y, Wei EP, Kontos HA, Suehiro E, Povlishock JT: Effects of delayed, prolonged hypothermia on the pial vascular response after traumatic brain injury in rats. J Neurosurg 2003, 99:899-906.

10. Kapp J, Mahaley MS Jr, Odom GL: Cerebral arterial spasm. 1. Evaluation of experimental variables affecting the diameter of the exposed basilar artery. J Neurosurg 1968, 29:331-338.

11. Bouzat $P$, Oddo M, Payen J-F: Transcranial Doppler after traumatic brain injury: is there a role? Curr Opin Crit Care 2014, 20:153-160.

12. De Riva N, Budohoski KP, Smielewski P, Kasprowicz M, Zweifel C, Steiner LA, Reinhard M, Fábregas N, Pickard JD, Czosnyka M: Transcranial Doppler pulsatility index: what it is and what it isn't. Neurocrit Care 2012, 17:58-66.

doi:10.1186/s13054-014-0693-8

Cite this article as: Donnelly and Czosnyka: The thermodynamic brain. Critical Care 2014 18:693. 